\title{
HESÍODO Y EL VALOR DEL OÎKOS: ESPACIO Y SUBJETIVIDAD
}

\author{
María Cecilia Colombani` \\ Universidad de Morón \\ Universidad Nacional de Mar del Plata
}

RESUMO: O plano do presente trabalho consiste em analisar as características que assume o lar em Os Trabalhos e os Dias, bem como a funcionalidade do mesmo no âmbito geral do poema. Sabemos que essa obra pode ser lida a partir de vários pontos de vista, sendo um deles a consideração do poema como um verdadeiro manual de conselhos práticos para o agricultor, que constitui a base da economia agrícola aldeã. Sem dúvida, por meio desta dimensão didática, surge a ideia do trabalho como algo regulador da constituição ética do homem. Se unirmos os dois elementos, trabalho mais agricultura, um modelo apropriado de interpretação é o valor do campo como articulador de ambos os tópicos. A referência à terra e ao espaço aberto que ela supõe é, sem dúvida, um elemento-chave da leitura do poema em termos topológicos ou espaciais.

PALAVRAS-CHAVE: trabalho; lar; lavrador; homem; constituição ética.

"En primer lugar, procúrate casa, mujer y buey de labor" (Trabajos y Días, 405)

^ceciliacolombani@hotmail.com 


\section{Introducción}

1 proyecto del presente trabajo consiste en analizar las características que toma el hogar en Trabajos $y$ Días, así como la funcionalidad del mismo en el marco general del poema. Sabemos que la obra puede leerse desde múltiples miradas siendo una de ellas la consideración del poema como un verdadero manual de consejos prácticos para el campesino, para el labrador que constituye la base de la economía agrícola de la aldea.

Sin duda, de la mano de esta dimensión didáctica, aparece la idea de trabajo como idea reguladora de la constitución ética del hombre. Si aunamos ambos andariveles, trabajo más agricultura, un modelo de instalación pertinente es el valor del campo como articulador de ambos tópicos. La referencia a la tierra y al espacio abierto que ella supone es, sin duda, un elemento clave de la lectura del poema en términos topológicos o espaciales.

El afuera donde se llevan a cabo los érga representa un tópos emblemático de la geografía que Hesíodo presenta; geografía de fuerte impronta subjetivante ya que el hombre se constituye como tal en el marco de la vida campesina, inscrita en la espacialidad que la tierra supone.

Así como la ciudad será el enclave subjetivante de los varones libres portadores de derecho en la Atenas Clásica, el campo resulta ser el tópos antropológico de la ecuación trabajo-agricultura. El énfasis puesto sobre el trabajo exterior que el cultivo de la tierra implica puede llevarnos a suponer que el mundo hesiódico está enteramente volcado hacia un "afuera" que predomina sobre un "adentro", de menor presencia en el poema.

Lejos de ello, nuestro propósito consiste en relevar la presencia del ôkkos y de la interioridad que el mismo supone, para descubrir un mundo humano que se juega en ambas dimensiones, idénticamente constitutivas. El hombre se consolida en el "entre" de ambas espacialidades, el afuera y el adentro, el mundo exterior del trabajo sobre la tierra y el mundo interior de la casa, donde también hay trabajo, tal como los versos devuelven.

Proponemos entonces una lectura de carácter antropológico indagando la coordenada del espacio para comprender un eje capital de la constitución del hombre. La diferencia entre el hombre y el animal gira en torno a la noción de albergue como noción antropológica. El hombre es hacedor de su albergue a partir de su concepción del espacio. Hesíodo está abriendo el juego de la reflexión filosófica en materia del universo humano. Los animales, incrustados en la naturaleza y fijados a ella sin posibilidad alguna de extrañamiento, carecen de albergue, de un espacio 
cargado de sentido. ${ }^{1}$

\section{El espacio íntimo - La casa-taller}

Hay una solidaridad insistente entre la casa y el mundo del trabajo, entre ese afuera y ese adentro que venimos persiguiendo. Lejos de constituir dos territorialidades nítidas, cerradas en sus propias esferas, sin conexión entre sí, hay en elemento que las pone en contacto, como una bisagra que determina cierto flujo entre lo que ocurre en el exterior y aquello que acontece en el interior: el trabajo. El trabajo es aquello que permeabiliza los tópoi porque la casa se convierte en una especie de taller donde se producen los elementos que luego juegan sus funciones en el afuera: "Fabrícate en casa todos los utensilios necesarios" (Trabajos y Días, 407-408).

La casa es el pues el espacio de una nueva dimensión del hombre, que no reduce su habilidad al cultivo de la tierra. Aparece un matiz artesanal en el varón, especie de tekhnites, capaz de resolver con sus manos aquellas necesidades que el trabajo demanda: "Construye trabajando en casa dos arados distintos, uno de una sola pieza y otro articulado, pues así será mucho mejor, y si rompes uno, puedes enganchar el otro a los bueyes" (Trabajos y Días, 433-435). ${ }^{2}$

Lo que se construye en el adentro se utiliza en el afuera, abriendo simbólicamente las compuertas que cierran al oîkos sobre su íntima espacialidad. La herramienta producida es el nexo entre una y otra geografía, extendiendo el hogar más allá de sus fronteras. La casa sale al exterior a través del útil producido en el interior.

A su vez, la casa-taller es el lugar para guardar las herramientas necesarias: "Llévate a casa un dental, buscándolo en la montaña o en el campo, de carrasca” (Trabajos y Días, 428-429). Se trata de la cama del

\footnotetext{
${ }^{1}$ Pensamos en la bibliografía clásica de la Antropología filosófica para esta afirmación. Tanto Max Scheler como Ernst Cassirer, Martin Buber o Emanuel Mounier, por nombrar sólo algunos autores clásicos de la disciplina, aluden a esta diferencia entre ambos tópoi, más allá de la noción legitimadora de tal diferencia o del marco teórico donde se instalen; se trate de un planteo espiritualista o de un abordaje materialista, el hombre parece estar transido por un plus que lo territorializa en otro espacio ontológico, ya sea en la línea de la continuidad o en la apuesta de la discontinuidad, el ser hombre entraña otras dimensiones, traducidas, precisamente, en la capacidad de gestar un kósmos humano.

${ }^{2}$ Tal como se aprecia existen dos tipos de arados, uno simple y otro compuesto; pero más allá de las particularidades y de la diferente tipología del instrumento, queda claro la necesidad de construir más de uno para que nunca se interrumpa el trabajo.
} 
arado, "el más resistente para arar con bueyes una vez que algún servidor de Atenea lo acople al timón fijándolo con clavos a la reja" (Trabajos $y$ Días, 430-432). ${ }^{3}$ Guardar y construir, la casa-taller no puede faltar en las previsiones del hombre prudente, ni en el conocimiento que la provisión de elementos exige: "A la sazón, ceba ya los bueyes de retorcidos cuernos que tienes en casa" (Trabajos y Días, 452-453). Ahora bien, aquello que se guarda ha implicado un arduo conocimiento, que aúna la relación entre el hacer y el saber hacer: "El hombre rico en imaginación habla de construirse un carro. iNecio!, no sabe que cien son las piezas de un carro que hay que preocuparse antes de hacer una casa" (Trabajos y Días, 455-458). La casa alberga la herramienta pero es también el espacio por donde circula el saber que lleva a la construcción. Es taller donde convive la habilidad manual de la construcción y la habilidad teórica de saber hacerlo.

\section{La casa-granero}

La casa opera también como un espacio de conservación de aquello que la tierra produce. Los vínculos entre el adentro y el afuera ahora se consolidan a partir de una función de almacenamiento que la materialidad de la casa posibilita. El trabajo exterior ha generado una buena cosecha, asociada simbólicamente a la idea de seguridad futura, que debe ser protegida en un espacio interior, afín a la protección de lo producido. El trabajo es la clave, "para que te aborrezca el Hambre y te quiera la venerable Deméter de hermosa corona y llene de alimento tu cabaña" (Trabajos $y$ Días, 300-301).

Con esta función de conservación quedan delineadas, a su vez, las características de ambos espacios, uno abierto, exterior, sin resguardo y poco proclive a la conservación y otro cerrado, íntimo, interior, resguardado, funcional a la práctica de acumulación.

Si en el tópico anterior, la casa salía al campo a través de las herramientas producidas, ahora el campo entra a la casa a través de lo que produce y debe ser resguardado. Nuevamente los tópoi se intersectan y muestran su complementariedad.

Este valor de la casa es altamente significativo ya que roza una preocupación fundamental para el hombre trabajador: conjurar la posibilidad del hambre como uno de los peores males. En este tópico, la casa resulta emblemática: "El que añade a lo que tiene evitará la quemazón del hambre; lo que hay dentro de casa no inquieta al hombre; es mejor

\footnotetext{
${ }^{3}$ La referencia a Atenea se vincula con la protección de la diosa a los artesanos, en este caso, a un carpintero, encargado del acople.
} 
tener dentro de casa; pues lo de afuera es dañino" (Trabajos y Días, 363-366). Los versos son reveladores en dos aspectos: en primer lugar, la seguridad que la casa brinda, pero, en segundo lugar, da cuenta también de un cierto matiz positivo del adentro frente al afuera. La casa parece representar la íntima seguridad que contrasta con la posibilidad del mal territorializada en el exterior.

La acumulación de los granos, asociada al trabajo arduo y a la previsión como marcas del hombre responsable, encuentra en la casa el espacio funcional a la exigencia antropológica. Sin el abrigo que la casa brinda sería imposible desarrollar la capacidad humana, altamente valorada, de considerar la relación tiempo-alimentación; guardar los granos en el espacio adecuado anticipa la posible tiranía del tiempo en materia de sustentabilidad. Quizás en esta línea deban leerse las recomendaciones de Hesíodo a Perses: "Pero tú preocúpate por disponer las faenas a su tiempo para que se te llenen los graneros con el sazonado sustento" (Trabajos $y$ Días, 307-308).

La casa es el espacio que posibilita el orden. La disciplina es la clave del trabajo y del éxito del mismo. Desde muchas aristas el poema devuelve la importancia de la disciplina en la vida de los hombres. En esta línea, la casa se convierte en aquello que permite el orden de lo almacenado $u$ obtenido por la labor: "Y a continuación, una vez que coloques ordenadamente todo el alimento dentro de casa, procúrate forraje y estiércol para que tengan en abundancia los bueyes y los mulos. Luego, por fin, deja que los esclavos relajen sus piernas y suelta los bueyes" (Trabajos y Días, 601- 602).

Idénticamente, el orden roza otra de las actividades que ubican al hombre en el exterior: el mar. Los elementos que posibilitan la navegación son pasibles del mismo orden que los frutos que brinda la tierra o los instrumentos que posibilitan su labranza. La casa entonces se inscribe en la actividad exterior constituyendo el espacio interior de la protección de los útiles. Representa entonces no sólo un albergue existencial, tema del próximo apartado, sino un recinto de resguardo del valor material de las cosas: "Guarda con orden en tu casa todos los aparejos en buenas condiciones y remienda las velas de la nave surcadora del ponto" (Trabajos y Días, 628-629).

\section{La casa-albergue}

La casa es espacio de reposo. Quizás sea esta dimensión la que más nítidamente de cuenta de la naturaleza interior y cerrada de la misma. La casa es el lugar del descanso reparador luego de la dura jornada de trabajo, aún frente a la inclemencia del Bóreas: "Anticípate a él y regresa a casa cuando termines el trabajo, no sea que algún día te cubra desde el cielo una 
nube sombría y deje húmedo tu cuerpo y empapados tus vestidos" (Trabajos y Días, 554-556). ${ }^{4}$ Sus puertas se cierran sobre su geografía interior para cerrar significativamente la dureza de las faenas exteriores, constituyendo la virtual imagen que proponemos, el símbolo del ciclo antropológico del hombre: trabajo-descanso. Un afuera que agota y un adentro que recibe en actitud acogedora es la nueva tensión que proponemos para pensar la complementariedad espacial.

En este caso la casa vuelve a rozar la ciclicidad humana y su materialidad es funcional a la díada en análisis. Descansar es el pasaporte a poder continuar con la tarea, la condición de posibilidad de la misma. Retorna, así, la misma imagen de la previsión que apareciera en el apartado anterior. Hay un espacio que parece solidarizarse íntimamente con la dimensión previsora, propia del hombre prudente: conservar el grano, conservar la energía a través del descanso adecuado. La casa es simbólicamente ese espacio donde la previsión toma cuerpo de êthos.

La casa es también el albergue de la "doncella de piel suave que dentro de la casa se queda junto a su madre, aún inexperta en los negocios de la muy dorada Afrodita" (Trabajos y Días, 520-522). Emblemático espacio de crianza que marcará claramente una dimensión de género, la casa es el espacio interior y cerrado de la doncella, su territorio natural antes de su contacto con los asuntos de Afrodita. Es allí donde se encuentra la joven, donde cumple su rutina y "lava su delicada piel, la unge con brillante aceite y va a recogerse al rincón más escondido de la casa en los días de invierno" (Trabajos y Días, 522-524). Espacio de recogimiento y albergue que pone a la casa en un lugar de protección.

\section{La casa-familia}

La casa constituye el albergue natural de la familia y el epicentro de su consolidación. Los consejos de administración familiar dan cuenta de la íntima solidaridad casa-mujer, hilvanando un vínculo indisoluble: "A madura edad llévate una mujer a tu casa" (Trabajos y Días, 695). ${ }^{5}$ Como

\footnotetext{
${ }^{4}$ El Bóreas es un viento que proviene de Tracia, del norte, hijo de Eos, la Aurora, y de Astreo, el titán. A su vez, es hermano de dos vientos, Céfiro y Noto, que Hesíodo presenta en Teogonía.

${ }^{5}$ La edad constituye un núcleo de preocupación intenso en los riesgos que de por sí acarrea el matrimonio. La edad madura equivale a los treinta años aproximadamente, lo cual genera una diferencia estatutaria con la esposa, quien debe pasar cuatro años de pubertad y luego casarse; la disimetría obedece seguramente a la permeabilidad de la joven a ser educada por el esposo. Sobre este tema puede verse Leduc, 1992:
} 
vemos, resulta el espacio simbólico aludido por Hesíodo a propósito de su preocupación en torno al matrimonio y a la familia, sobre todo a la mujer con la ambigua consideración que la misma ostenta al interior del corpus. Constituye entonces el territorio emblemático de la mujer, asociada a la funesta descendencia de Pandora; la cobija en su interior, reforzando la improductividad propia de sus marcas identitarias. Si el trabajo es eminentemente una gesta exterior, la no productividad femenina la territorializa en el interior de la casa, "siempre pegada a la mesa" (Trabajos y Días, 704) y consumiendo el granero en actitud predatoria. La pintura de la mujer al respecto es ilustrativa: "Que no te haga perder la cabeza una mujer de trasero emperifollado que susurre requiebros mientras busca tu granero" (Trabajos y Días, 373-375).

La permeabilidad entre los espacios que venimos abordando sólo se logra a través de las marcas de la buena esposa, ya que el trabajo la lleva del adentro al afuera, del interior al exterior. El trabajo es siempre la condición de posibilidad de abrir las puertas que clausuran la vida al interior del ô̂kos. Ese tránsito de una actitud "naturalmente" pasiva a otra activa sólo es posible a partir de una tarea pedagógica que el marido lleva a cabo.

Si bien hemos demostrado que la interioridad de la casa no es sinónimo de pasividad, ya que hemos relevado acciones que se cumplen en su interior, la permanencia improductiva de la mujer en la casa, específicamente asociada a la mesa, refuerza el imaginario de la pasividad doméstica frente a la actividad extramuros.

La casa es también el lugar del respeto. Casa y familia hilvanan un tapiz vincular que no puede mancillarse con actitudes que ofendan la materialidad simbólica de la primera y la honra que le corresponde a la segunda: "No te dejes ver con los genitales manchados de semen dentro de tu casa junto al hogar, sino evítalo" (Trabajos y Días, 733-734). Los escoliastas han interpretado que el acto sexual mancha al hombre de impureza; por ello no debe acercarse al centro mismo de la casa, al lugar emblemático, el hogar, donde se rinde culto a los Bienaventurados.

\section{La casa-vínculo}

La casa parece ser un espacio de construcción vincular; no el único pero sí el que guarda peculiares características que pueden ubicarse en un registro de marcada familiaridad y de cierta intimidad. Cuando el poeta se refiera a la casa ubica en ella o bien el vínculo del hombre consigo mismo,

“¿Cómo darla en matrimonio? La novia en Grecia, siglos IX-IV a. C.”; en el texto aparece con claridad el dispositivo matrimonial griego. 
o bien el vínculo con la esposa, lo cual habla precisamente de la intimidad aludida. La casa cobra protagonismo a partir de un vínculo familiar que el poeta sugiere: "Procura tener un solo hijo, para conservar intacto tu patrimonio; pues así la riqueza crecerá dentro de tu casa" (Trabajos y Días, 376-377). La casa es el tópos que alberga la riqueza; su espacialidad cerrada contiene la prosperidad y evita el derroche.

Otros espacios también dan cuenta de la construcción vincular. Vínculos del "afuera" frente a los vínculos del "adentro" que la casa implica como espacio íntimo y celular de tejido relacional. El plano material del trabajo genera esos vínculos extramuros. El labrador y sus criados, el labrador y sus esclavos, copartícipes en la relación de trabajo constituyen la trama de un tejido de relaciones que trasciende el universo íntimo del hogar en torno a los trabajos de otoño: "Que los siga un hombre fuerte de unos cuarenta años después de desayunar un pan cuarteado de ocho trozos" (Trabajos y Días, 441-442). Este trabajo es complementario de otro que genera, a su vez, un vínculo de complementariedad laboral: "Otro no más joven que éste es el mejor para volear las semillas y evitar su acumulación” (Trabajos y Días, 445448). El campo como espacio de la labor genera un vínculo complementario que, como siempre, mira a la prosperidad del trabajo.

Otro pasaje devuelve también la construcción de vínculos de trabajo pero también de subordinación estatutaria: "Cuando ya se muestren a los mortales los primeros días de la labranza, poned entonces manos a la obra, junto los criados y tú, arando la tierra seca y húmeda en la época de labranza y ganando tiempo muy de mañana, para que se llenen de frutos tus campos" (Trabajos y Días, 458-462). El trabajo compartido genera de hecho vínculos que refuerzan la excelencia del trabajo a partir de la colaboración entre actores de distinto rango, el labrador y sus criados.

El vínculo de colaboración entre miembros que constituyen el patrimonio de la casa, retorna a propósito de la tarea del pequeño esclavo: "Detrás el pequeño esclavo dará trabajo a los pájaros enterrando las semillas con la azada" (Trabajos y Días, 469-471). Los vínculos generan distintas relaciones de edad y de tareas, pero hablan del espacio abierto como tópos de consolidación de círculos que impactan sobre la prosperidad de la casa, a través del beneficio del trabajo.

El ágora es otro espacio del vínculo "exterior". La relación con los pares, el vecino, el amigo, el pariente, los reyes van hilvanando un plexo de vínculos que se juegan en el afuera.

\section{Casa, campo, ágora - las fronteras se desdibujan}

Nuestro trabajo ha dado muestras de la complementariedad entre la casa como espacio interior y el espacio exterior, el tópos del afuera, 
predominantemente, representado por el campo como el espacio abierto donde se juega el trabajo del labrador. No obstante, no se trata del único afuera que el poema refiere. La referencia a la navegación abre otro afuera, el mar; un puro espacio exterior, móvil y acuoso, de difícil tránsito.

Otra geografía extramuros que el poema sugiere es el ágora, analizada, en el apartado precedente, como espacio de la lucha política que la aldea sugiere en su quehacer cotidiano.

Nos gustaría pensar el denominador común entre tres de estos espacios: la casa, el campo y el ágora. La primera nota ha sido la condición de espacios donde es posible el vínculo intersubjetivo. Complementaria de esta dimensión es su condición de espacios civilizados, de tópoi transidos por la noción de cultura como aquello que distingue específicamente al hombre y lo aleja de la lógica imperante entre las bestias.

Quizás la referencia al pleito que sostiene con su hermano sea la pintura más nítida de la complementariedad de los espacios: la casa, el campo y el ágora como tópoi culturales; Hesíodo opone el ágora, territorio de la contienda y espacio afín a Perses, "preocupado por acechar pleitos en el ágora" (Trabajos y Días, 30), y la casa, lugar donde debe hallarse el sazonado sustento, "el grano de Deméter, que la tierra produce" (33), merced al trabajo desplegado en el campo.

La casa es el micro espacio de instalación antropológica con todas las pautas que ello implica; el campo ha dejado de ser la tierra baldía, inculta, para ser la geografía próspera y fecunda que devuelve, trabajo mediante, el fruto de la diosa; el ágora es el espacio emblemático de un signo de cultura: el derecho a la palabra que circula, el espacio del litigio entre hermanos, el tópos de la denuncia a los dorophágoi.

\section{Conclusiones}

Tres espacios de corte diferente, de distinto registro en la tensión exterior-interior, pero emparentados entre sí a partir de una nota común: la civilidad de su materialidad.

De lo micro a lo macro las fronteras territoriales se disuelven a la hora de relevar la espacialidad en términos antropológicos: tres lugares de construcción del vínculo, con los íntimos, con la tierra y sus trabajadores, con los aldeanos.

Del adentro al afuera los espacios pierden por un momento su singularidad específica para constituir la materialidad de un espacio antropológico que trasciende las connotaciones geográficas.

Del espacio en términos territoriales al espacio en términos humanos, la casa, el campo y el ágora constituyen las geografías subjetivantes que delinean la constitución del hombre como animal simbólico, capaz de construir sentido en esos tópoi. 
La casa, el ágora y el campo constituyen los espacios donde se inscribe la capacidad poiética del hombre como hacedor de cultura; espacios, a su vez, íntimamente vinculados al trabajo como actividad subjetivante. He aquí otro punto de intersección que desdibuja las fronteras entre el adentro y el afuera. Espacios de inscripción de cultura, el territorio, interior o exterior, es el soporte material de la constitución antropológica. Así, la noción de mundo nunca implica una noción territorial, de registro topológicoespacial, sino un concepto antropológico, de matriz significativa. El mundo es "el lugar en el mundo", el domicilio existencial del hombre en tanto hacedor de cultura, y explicita la relación del hombre con la naturaleza como primer enclave antropológico.

Hesíodo contribuye a ordenar no sólo el mundo divino con su genealogía, sino también el mundo humano, haciéndolo pasar por el eje del trabajo como actividad constitutiva del hombre. Ocurre que es por el trabajo que el hombre puede darse un lugar en el mundo. El trabajo es así un rasgo humano y civilizador, cuyo tratamiento vuelve a poner a Hesíodo en un registro didáctico. Trabajos y dias, como afirma Fontenrose, "is both a plea for justice and a gospel of work". " El poeta enseña cómo se alcanza la dignidad del hombre. En realidad, se están sentando las bases para la constitución de una pólis justa y organizada, lo cual supone una intensa preocupación por el vínculo trabajo-êthos en tanto manera de vivir. ${ }^{8}$

\section{Referencias}

BUBER, M. Yo y tú. Trad. Horacio Crespo. Buenos Aires: Nueva Visión, 1974.

CASSIRER, E. Antropología filosófica. Trad. Eugenio Ímaz. México: FCE, 1974.

COLOMBANI, M. C. Hesíodo: una introducción crítica. Buenos Aires: Santiago Arcos, 2005.

DETIENNE, M. Los maestros de verdad en la Grecia Arcaica. Trad. Juan José Herrera. Taurus: Madrid, 1986.

FONTENROSE, J. Work, justice, and Hesiod's five ages. Classical philology, Chicago, vol. LXIX, n 1, p. 1-16, 1974.

\footnotetext{
${ }^{6}$ Cf. Heidegger, 1997, p. 79.

${ }^{7}$ Cf. Fontenrose, 1974, p. 5.

${ }^{8}$ Coincidimos con la postura de Judet de La Combe y Lernould, 1996, p. 302, cuando sostienen que le travail apparaît comme l'activité qui définit l'homme essentiellement. En efecto, la disimetría ontológica entre ambos planos, el humano y el divino, hace que el trabajo se defina desde un registro que va más allá de su dimensión funcional.
} 
HEIDEGGER, M. Ser y tiempo. Trad. José Gaos. Santiago de Chile: Editorial Universitaria, 1997.

HESÍODO. Teogonía; Trabajos y días; Escudo. Trad. Aurelio Pérez Jiménez. Barcelona: Editorial Planeta/ De Agostini, 1997.

HESÍODO. Obras y fragmentos. Trad. Aurelio Pérez Jiménez. Gredos: Madrid, 2000. JUDET DE LA COMBE, P.; LERNOULD, A. Sur la Pandore des «Travaux»: esquisses. En: BLAISE, F.; JUDET DE LA COMBE, P.; ROUSSEAU, P. (org.). Le métier du mythe: lectures d'Hésiode - vol. 16. Paris: Presses Universitaires du Septentrion, 1996, p. 301-313.

LEDUC, C. ¿Cómo darla en matrimonio? La novia en Grecia, siglos IX-IV a.C. En: DUBY, G.; PERROT, M. Historia de las mujeres, 1 - la Antigüedad. Trad. Marco Aurelio Galmarini. Madrid: Taurus, 1992, p. 251-313.

LIÑARES, L. Hesiodo: Teogonía; Trabajos y Días. Edición bilingüe. Buenos Aires: Losada, 2005.

MOUNIER, E. El personalismo. Trad. Aída Aisenson, Beatriz Dorriots y León Rozitchner. Buenos Aires: EUDEBA, 1972.

SCHELER, M. El puesto del hombre en el cosmos. Trad. José Gaos. Buenos Aires: Losada, 1972. 
